\title{
Risk-hedging using options for upgrading investments in mobile networks
}

\author{
Frédéric Morlot, Salah Eddine Elayoubi, Thomas Redon \\ Orange Labs \\ 38-40 rue du Général Leclerc \\ 92794 Issy-Les-Moulineaux, France \\ frederic.morlot@polytechnique.org, salaheddine.elayoubi@orange.com, thomas.redon@polytechnique.org
}

\begin{abstract}
In this paper, we illustrate how a mobile network operator can plan an upgrading investment to anticipate explosions of the traffic demand, taking into account the expected generated profit and the customers satisfaction. The former parameter grows with the demand, whereas the latter sinks if the demand is too high as individual Quality of Service (QoS) may collapse due to capacity saturation problems. In addition to that, as the equipment price decreases with time, it may be interesting to wait rather than to invest at once. Taking into account this trade off, we propose a real option strategy to hedge against the risk that the investment has to take place earlier than expected. At last, we price this option with a backward dynamic programming approach, using recent improvements based on least-squares estimations.
\end{abstract}

\section{INTRODUCTION}

Today, it is expected that the mobile data traffic will be significantly growing. To face these soaring volumes of data to be transferred, mobile operators must periodically upgrade their equipments to offer higher throughputs and avoid QoS (Quality of Service) problems. However as the demand does not increase steadily and must be considered as partly random, the upgrading investment date is difficult to be forecast.

In this article, we consider upgrade investments in a telecommunication network, with an application to Beyond 3G wireless systems. In this context, when the traffic increases, the operator naturally increases its profit until the demand approaches the limiting capacity of the network. In this case, the network starts experiencing saturation, throughput and QoS problems, so the operator's profit may fall. The operator must then upgrade its network to increase the capacity. An example of an upgrading means consists in adding a frequency carrier. The operator then faces the following trade-off:

- The later the investment, the lower individual throughputs and customer satisfaction. Permanent non-satisfaction will result into churn and additional loss of profit.

- The sooner the investment, the more expensive the costs of upgrade elements.

This article aims at resolving analytically this trade-off. Throughout the paper, we propose a general methodology and illustrate it on the practical example of HSDPA networks. We first derive analytical value for operator's profit, taking into account randomness of the rising demand, and decrease of network element costs according to time. We second introduce a real options method to hedge against the risk that demand evolves in an unexpected way leading to a premature investment decision or a too late one. To perform that, we introduce an American call that allows its owner (the mobile operator) to buy an equipment at a fixed price, possibly less than the real one, until a maturity date. Given the profit analytical model and the option's parameters, we propose a dynamic programming method to price the option. At the same time, we obtain the expected best investment date.

\section{RELATED WORK}

Recently, the real options framework [1] has been widely used for evaluating investments under uncertainty when timing is a critical issue and the infrastructure project requires the commitment of huge costs up front. The real options approach evaluates a firm's opportunity to invest in a real, partially or completely irreversible, project, in uncertain future environment by evaluating the managerial flexibility as a financial option [2]. The first applications of real options where in natural resource investments as in [3] where the authors valued a Gold Mine as an option. Afterwards, the real options method were used for evaluating transportation projects [4], sustainable development investments [5][6], and even intellectual property rights [7], etc. In these contexts, uncertainty can come from the project revenue [3], from the demand [4][5], or from $R \& D$ efforts that may reduce the investment costs [6][8].

In the field of telecommunications, real options are classically used for evaluating strategic decisions like regulation of the telecommunication market [9], definition of tariffs for operators [10], or pricing of spectrum licenses [11]. This work focuses on a less macroscopic application, but that is of high importance for operators: deploying mobile network infrastructures for an already licensed system, in order to respond to the progressive increase of customer demand. Only few works was interested by applying real options on this field. For instance, d'Halluin presented a method to determine the best investment date in a fixed [12] or a wireless [13] network, based on real options and a simple network capacity model. To the best of our knowledge, this work is the first that considers option's pricing for capacity investments in mobile networks, 
based on realistic network capacity models, and that proposes both optimal timing and risk hedging for the investments.

The remainder of this paper is organized as follows: in a first section, we build an analytical model of the operator's profit and illustrate it on an HSDPA network. Then we introduce in section II an American option to hedge against the risk aforementioned. We define the underlying asset and the option's payoff. To price the option we use a riskneutral approach, whose mathematical justification lies in the appendix. In section III, we show how dynamic programming can help solving the pricing problem, and the best investment date problem as well. In section IV, we present the numerical results before concluding the paper.

\section{THE BASIC MODEL: OPERATOR'S PROFIT AND INVESTMENT COST}

In this section, we calculate the operator's profit, that will be used next in evaluating the investment.

\section{A. Traffic Demand}

The operator profit depends on the amount of data flowed by the network. The traffic is generally composed of real time (voice, streaming) as well as elastic traffic. Real time traffic is measured in minutes of communication per hour per cell, generally translated into Erlang/cell. For elastic traffic, this demand is expressed in Mbits/sec/cell (aggregated traffic demand from all clients in a typical cell). We denote the overall traffic (real-time or elastic) by $X_{t}$, where the time $t=0,1 \ldots$ varies discretely.

To model the evolution of $\left(X_{t}\right)_{t \in \mathbb{N}}$, let us consider it as the daily sampling of a continuous stochastic process $\left(\tilde{X}\left(t, W_{t}\right)\right)_{t \in \mathbb{R}^{+}}$. As many random phenomena related to a social behavior (e.g. [5]), we assume that $\tilde{X}\left(t, W_{t}\right)$ is a geometric Brownian motion:

$$
\tilde{X}\left(t, W_{t}\right)=x_{0} \mathrm{e}^{\left(\alpha-\sigma^{2} / 2\right) t+\sigma W_{t}}, \quad t \in \mathbb{R}^{+},
$$

where $W_{t}$ is a standard Brownian motion, $\alpha$ is the trend of the demand and $\sigma$ is its volatility.

\section{B. Customers satisfaction and operator's profit}

In this section, we define the relationship between the traffic, the capacity, the QoS and the profit. Let the capacity of a typical cell of the network be equal to $C$. The QoS perceived by users depends on the load, defined by the ratio between the traffic and the capacity. In all cases, the QoS is a function $Q_{t}\left(X_{t}, C\right)$ that decreases when the traffic increases, and that increases when the capacity is higher. This QoS is generally expressed in throughput for elastic traffic and access probability for real-time traffic.

Now we can compute the customer satisfaction, which can reasonably be supposed to depend on the QoS. For elastic traffic, subjective satisfactions have been shown to be more sensitive to small variations at low throughputs than at high throughputs. Enderlé and Lagrange proposed in [17] to model the customer satisfaction as a negative exponential function of the throughput:

$$
H_{t}\left(X_{t}, C\right)=\mathrm{e}^{-\beta / Q_{t}\left(X_{t}, C\right)} .
$$

For example, $\beta$ can be chosen as $\beta=\log (2) \cdot q_{1 / 2}$, where $q_{1 / 2}$ is the throughput value ensuring a satisfaction of $50 \%$.

For voice service, QoS is an access rate (probability of not being blocked), so users that have access to the service are fully satisfied, while the others are completely unhappy. Let $a\left(X_{t}, C\right)$ be the access rate when the traffic intensity is equal to $X_{t}$ and the cell capacity is $C$, the satisfaction is thus directly equal to this access rate: $H_{t}\left(X_{t}, C\right)=a\left(X_{t}, C\right)$.

Finally, the operator's profit can be calculated. If $\delta$ is the transfer price (say in \$/Mbit for elastic traffic or in \$/Erlang for real-time traffic), the gross daily profit per cell is given by:

$$
\pi^{\text {gross }}=\delta X_{t},
$$

However the gross profit should be weighed by the customer satisfaction to account for the quality of the communications. The net profit is thus calculated as the product of $\pi^{\text {gross }}$ by $H_{t}$ :

$$
\pi_{t}\left(X_{t}\right)=\delta X_{t} H_{t}\left(X_{t}, C\right)
$$

\section{Upgrading Investment}

When traffic increases, QoS will surely degrade, and so the satisfaction $H_{t}$. If no upgrading action is taken, the profit will progressively tend to zero. Once the operator decides to upgrade, he can install additional equipments offering higher capacity (e.g., by adding transmitters operating on different frequency bands). In such a case we obtain a higher value $C^{\prime}$ of the capacity. The profit becomes:

$$
\pi_{t}^{\prime}\left(X_{t}\right)=\delta X_{t} H_{t}\left(X_{t}, C^{\prime}\right)
$$

On the other hand, the upgrading (equipment) cost is a decreasing function of time (due, for instance, to the R\&D progress or the serialization in the manufacturing chain). In this paper we assume it decreases exponentially $(K(t)=$ $K_{0} \mathrm{e}^{-\epsilon t}$ ), where $\epsilon$ is the depreciation rate.

\section{Total Profit}

Let us introduce date $T$, at which the investment becomes obsolete (in other words, the proposed investment cannot be undertaken after $T$ ). If we denote the investment date by $t_{0}$ $\left(0<t_{0}<T\right)$, the total profit $\Pi_{T}\left(t_{0}\right)$ actualized at $t=T$ is:

$$
\Pi_{T}\left(t_{0}\right)=\sum_{t=0}^{t_{0}-1} \mathrm{e}^{\zeta(T-t)} \pi_{t}+\sum_{t=t_{0}}^{T} \mathrm{e}^{\zeta(T-t)} \pi_{t}^{\prime}
$$

where $\zeta$ is the actualization rate. For simplicity, we assume that $\zeta$ is constant during the period $[0, T]$. 

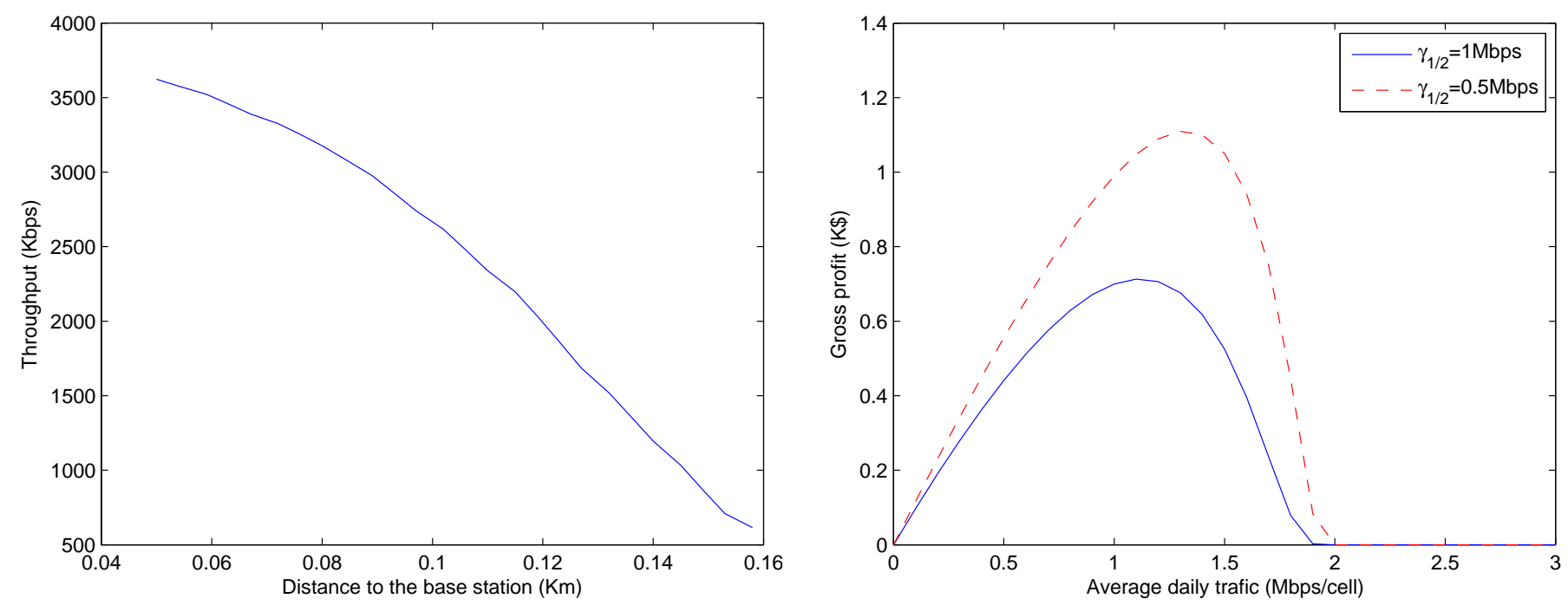

Fig. 1. Peak data rate versus distance to the base station. The system is HSDPA in a dense urban environment.

\section{E. Application to Beyond $3 G$ networks}

In this section, we show how to calculate the QoS and the profit for elastic traffic in beyond $3 \mathrm{G}$ systems, including HSDPA, 3GL LTE and WiMAX systems. These systems are characterized by a throughput that depends on the position of the users in the cell: Cell edge users have lower throughputs than cell center ones, as illustrated in Figure 1. As far as we are considering elastic services, users with degraded throughput will stay longer in the cell and contribute more to the cell load. It has thus been shown in [14] that the maximal capacity of the cell is equal to the harmonic mean of the throughput. Let $C$ be this maximal capacity.

The load of the cell is thus equal to the ratio between the offered traffic and the capacity: $\rho_{t}=\frac{X_{t}}{C}$. Using simple processor sharing arguments, it can be shown [14] that, provided that the load is less than 1 , the average flow throughput is:

$$
\bar{Q}_{t}\left(X_{t}, C\right)=C\left(1-\rho_{t}\right)=C\left(1-\frac{X_{t}}{C}\right)=C-X_{t}
$$

However, when the load exceeds one, the throughput of all users falls to 0 . The

$$
Q_{t}\left(X_{t}, C\right)=\left(C-X_{t}\right)^{+},
$$

where $x^{+}=\max (x, 0)$.

The satisfaction is thus calculated by:

$$
H_{t}=\mathrm{e}^{-\beta /\left(C-X_{t}\right)^{+}} \text {. }
$$

and the gross daily profit per cell is given by:

$$
\pi^{\text {gross }}=\delta \min \left(X_{t}, C\right),
$$

where $\delta$ is the transfer price (say in \$/Mbit). Finally, the net profit is calculated as the product of $\pi^{\text {gross }}$ by $H_{t}$ :

$$
\pi^{n e t}=\delta \min \left(X_{t}, C\right) \mathrm{e}^{-\beta /\left(C-X_{t}\right)^{+}} .
$$

Fig. 2. Daily Profit generated by demand. $C=2 \mathrm{Mbits} / \mathrm{sec} / \mathrm{cell}$, $\delta=0.1 \$ / \mathrm{Mbit}, \mu=14000 \mathrm{sec}$. Two values of $\beta$ are considered, corresponding to target throughputs $\gamma_{1 / 2}=1 \mathrm{Mbit} / \mathrm{sec}$ and $0.5 \mathrm{Mbit} / \mathrm{s}$. It can be observed that the profit is better when users are satisfied with a smaller throughput, but it always collapses when the traffic approaches the maximal capacity.

If $H_{t}=0$, i.e. if the cell is saturated, the net profit is null. If the satisfaction is maximal, i.e., $H_{t}=1$, the net profit is equal to the gross profit (1). To sum up we have:

$$
\begin{array}{ll}
\pi_{t}=\delta X_{t} \mathrm{e}^{-\beta /\left(C-X_{t}\right)} & \text { if } X_{t}<C \\
\pi_{t}=0 & \text { otherwise. }
\end{array}
$$

Intuitively, as the demand rises, $X_{t}$ will increase as will the profit (Fig. 2). Then, the profit will decrease because the unsatisfaction effect becomes dominant.

\section{RISK-HEDGING USING AN AMERICAN OPTION}

\section{A. Externalizing the financial risk}

As shown above, there is a trade off between the growth of the demand (encouraging to invest) and the depreciation of the equipment cost (encouraging to wait). Then the risk is to be led to invest while the equipment is still expensive. In this section we show how to hedge against this risk using an American option. This option, acquired from a third party like a bank, gives us the right to buy the equipment at price $K^{\star}$ instead of $K(t)$, until date $t^{\star}=t\left(K^{\star}\right)$. Let us recall that the operator has the right but not the obligation to exercise this option, but has to pay in return a premium to the bank, denoted by $P$. If he has to invest before date $t^{\star}$, he will exercise the option, give $K^{\star}$ to the equipment provider and the bank will pay the difference. Otherwise, he will not exercise the option and he will lose the premium, but he still can invest. In this section we will try to answer the two following questions:

- when is this option going to be exercised ?

- how much does it cost (i.e. calculate $P$ ) ? 


\section{B. Introducing the American option}

When is the option going to be exercised ? It depends on the additional profit expected from investing to upgrade the network: at least, this additional profit has to be greater than $K^{\star}$. At date $t$, it can be expressed as follows:

$$
S_{t}=\mathbb{E}\left[\int_{t}^{T} \mathrm{e}^{-\zeta(s-t)}\left(\pi^{\prime}\left(s, W_{s}\right)-\pi\left(s, W_{s}\right)\right) d s \mid \mathcal{F}_{t}\right] .
$$

Facing the decision to invest or not, the operator's strategy is to compare the profit realized if investing with the value of waiting, typically to check that the traffic is not going to decrease unexpectedly which would make the upgrading expenditure a sunk cost. This appears to be the classical problem of finding the exercise strategy for an American option, with the following features:

- $t^{\star}$ as the option's maturity

- $K^{\star}$ as the exercise price or strike

- $S_{t}$ as the underlying asset

- $\left(S_{t}-K^{\star}\right)^{+}$as the option's payoff, denoted by $Z(t)$ :

$$
Z(t)=\max \left\{S_{t}-K^{\star}, 0\right\}
$$

\section{Pricing of the American option}

The resolution of this problem appeals to classical stochastic theory and the risk-neutralization approach ([19][15]).

1) Preliminaries: to detail this approach, let us introduce two progressively measurable processes $\mu_{t}$ and $\kappa_{t}$, respectively the expected total return on the asset and its volatility:

$$
d S_{t} / S_{t}=\mu_{t} d t+\kappa_{t} d W_{t}
$$

along with the market price of risk:

$$
\theta_{t}=\kappa_{t}^{-1}\left(\mu_{t}-\zeta\right)
$$

We obtain expressions of $\mu_{t}, \kappa_{t}$ and $\theta_{t}$ in the appendices VII-A to VII-C, where we show that:

$$
\theta_{t}=-\frac{\pi^{\prime}\left(t, W_{t}\right)-\pi\left(t, W_{t}\right)}{\frac{\partial v}{\partial x}\left(t, W_{t}\right)}
$$

with:

$v(t, x)=\int_{t}^{T} \mathbb{E}\left[\mathrm{e}^{-\zeta(s-t)}\left(\pi^{\prime}\left(s, W_{s}\right)-\pi\left(s, W_{s}\right)\right) \mid W_{t}=x\right] d s$

Note that applying the risk-neutralization approach will also require Novikov's condition (see [16], page 65), stating that:

$$
\mathbb{E}\left[\exp \left(\frac{1}{2} \int_{0}^{T} \theta_{t}^{2} d t\right)\right]<+\infty
$$

In the appendix VII-D, we show that Novikov's condition is verified in our specific case.
2) The risk-neutralization approach: under this condition, let $\mathcal{S}\left(\left[t, t^{\star}\right]\right)$ be the set of stopping times with values in $\left[t, t^{\star}\right]$ and define the following process known as the Snell envelope:

$$
Y_{t}=\sup _{\tau \in \mathcal{S}\left(\left[t, t^{\star}\right]\right)} \mathbb{E}_{\mathbb{Q}^{*}}\left[\mathrm{e}^{-\zeta \tau} Z(\tau) \mid \mathcal{F}_{t}\right]
$$

Here, $\mathbb{Q}^{\star}$ is the risk-neutral probability, whose density w.r.t. $\mathbb{P}$, the historical probability, is:

$$
\frac{d \mathbb{Q}^{\star}}{d \mathbb{P}}=\exp \left(-\frac{1}{2} \int_{0}^{t^{\star}} \theta_{s}^{2} d s-\int_{0}^{t^{\star}} \theta_{s} d W_{s}\right) .
$$

In fact, since we will have to simulate trajectories of the asset beyond date $t^{\star}$ (until date $T$ ), we will rather choose the probability $\mathbb{Q}$, whose density w.r.t. $\mathbb{P}$ is:

$$
\frac{d \mathbb{Q}}{d \mathbb{P}}=L_{T}=\exp \left(-\frac{1}{2} \int_{0}^{T} \theta_{s}^{2} d s-\int_{0}^{T} \theta_{s} d W_{s}\right) .
$$

Note that $\mathbb{Q}$ is indeed a probability measure, since $\mathbb{E}_{\mathbb{P}}\left[L_{T}\right]=1$, as $\theta_{t}$ verifies Novikov's condition (see previous paragraph). Note also that $\mathbb{Q}^{\star}$ is the restriction of $\mathbb{Q}$ to $\mathcal{F}_{t^{\star}}$ (see [15], Theorem 9.1.2.), so that (11) still holds with $\mathbb{Q}$ if $t \leq t^{\star}$. Then the premium of the option at any time $t \in\left[0, t^{\star}\right]$ is given by [19]:

$$
\Pi_{t}=\mathbb{E}_{\mathbb{Q}}\left[\mathrm{e}^{-\zeta(\tau(t)-t)} Z(\tau(t)) \mid \mathcal{F}_{t}\right]
$$

where $\tau(t)$ is the solution of the maximization in (11). $\tau(t)$ is interpreted as the optimal exercise strategy of the option calculated at date $t^{1}$.

\section{THE DYNAMIC PROGRAMMING SOLUTION}

As stated above, the problem is to find the stopping time maximizing the option's payoff under risk neutrality (Eqn. (11)). However, it is impossible to compute $Z(t)$ analytically, so we make use of a dynamic programming approach, as in [20]. We recall that it consists in dividing the problem into two binary decisions at the final date $t^{\star}$ : the "immediate" one and its generated value, and the "delaying" one and its continuation value. Then moving backward, and repeating the same binary decision, we obtain the expected optimal time which lies in an expected interval in which the investment should be undertaken [8]. We must then, at each moment, find two different values: the option's payoff in case of investment and the continuation value in case of waiting.

\section{A. Monte-Carlo simulations to generate the underlying asset}

Calculating $S_{t}$ involves a complex integration (Eqn. (6)) that cannot be performed analytically. We then use Monte-Carlo simulations as follows:

$$
\begin{aligned}
& { }^{1} \text { Note that if the option were a European option, the price at date } t \text { would } \\
& \text { be: } \\
& \qquad \Pi_{t}=\mathbb{E}_{\mathbb{Q}}\left[\mathrm{e}^{-\zeta\left(t^{\star}-t\right)} Z\left(t^{\star}\right) \mid \mathcal{F}_{t}\right]
\end{aligned}
$$

(see [15] page 65). But here, our option is an American option, so we have to generalize this result and to use the Snell envelope. 
- first we compute $v(t, x)$ with Eqn. (9) for $t \in\left[0, t^{\star}\right]$ and $x \in\left[w_{\text {min }}, w_{\text {max }}\right]^{2}$.

- we then make time discrete: $t=t_{0} \ldots t_{N}$ with $t_{0}=0$ and $t_{N}=t^{\star}=N \delta t$. After that we simulate $J$ trajectories of $S_{t}$ under $\mathbb{Q}$ : the $j$-th trajectory is denoted by $\left(S^{j}\right)$ and has the value $S_{n}^{j}$ at time $t_{n}=n \delta t$. More precisely, we simulate (under $\mathbb{Q}$ ) $J$ trajectories of the historical Brownian $\left(W^{j}\right)^{3}$, and then we compute $S_{n}^{j}=v\left(t_{n}, W_{n}^{j}\right)$ by interpolating $v(t, x)$. This is far more efficient than computing directly the integral, especially if we want to simulate a large number of trajectories, since we do not have to compute $v$ each time again.

\section{B. Continuation value and decision tree algorithm}

At time $t^{\star}$, the operator invests if $Z_{N}>0$. More generally, at a time $t_{n}<t^{\star}$, the operator has two alternative choices: either invest now and get $Z_{n}$, or wait and get the expected continuation value, denoted by $C_{n}$. The generated cash-flow is then given by:

$$
F_{n}=\max \left\{Z_{n}, C_{n}\right\} .
$$

We already know $Z_{n}$ by (7). As for $C_{n}$, we use the Least Squares Monte-Carlo (LSM) approach defined by Longstaff and Schwartz [20]. This approach consists in writing the expected continuation value $C_{n}$ as a general function of $S_{n}$ (in our case we took a 2-degree polynom), taking information from the $J$ cash-flows at $t_{n+1}$ and using the fact that:

$$
C_{n}(S)=\mathrm{e}^{-\zeta \delta t} \mathbb{E}\left[F_{n+1} \mid S_{n}=S\right],
$$

where $F_{n+1}$ is the (random) cash-flow of the option at $t_{n+1}$. To obtain recursively $C_{n}$, we can write the following algorithm:

- at $t_{N}$, for each trajectory $j=1 \ldots J$, calculate the cashflow $F_{N}^{j}=Z_{N}^{j}$.

- move one period back to $t_{N-1}$. For each $\left(S^{j}\right)$, check if the option is "in the money", i.e. if $Z_{N-1}^{j}>0$. If it is the case, calculate the continuation value $C_{N-1}^{j}$ using the cash-flow if investment is delayed: $C_{N-1}^{j}=\mathrm{e}^{-\zeta \delta t} F_{N}^{j}$. Estimate then the general expression of $C_{N-1}(S)$ by the LSM algorithm. This consists in regressing the found values $C_{N-1}^{j}$ on a constant, $S$ and $S^{2}$, as in [20] (see appendix VII-E). Let us denote the estimated expression by $\hat{C}_{N-1}(S)$. The estimated cash-flow at $N-1$ is then given by:

$$
F_{N-1}^{j}=\max \left\{Z_{N-1}^{j}, \hat{C}_{N-1}\left(S_{N-1}^{j}\right)\right\} .
$$

If it is optimal to exercise at $t_{N-1}$, then by convention $F_{N}^{j}$ becomes 0 (because the option can only be exercised once).

- for each time $t_{n}$, repeat the same process until $n=0$.

\footnotetext{
${ }^{2}$ to bind efficiently the Brownian motion, see Appendix VII-D.

3 to perform that, assuming that the probability of our random generator is $\mathbb{Q}$, we simulate a standard Brownian motion $\left(W_{t}^{\mathbb{Q}}\right)$, and then using Girsanov's

\begin{tabular}{|c|c|}
\hline \multicolumn{2}{|c|}{ 2. for $j=1 \ldots J$, put $F_{N}^{j}=Z_{N}^{j}$} \\
\hline \multicolumn{2}{|c|}{ 3. for $n=(N-1) \ldots 1,0$ : } \\
\hline \multicolumn{2}{|c|}{$\begin{array}{l}\text { 3.1. for } j=1 \ldots J \text {, calculate } Z_{n}^{j} \text { : } \\
\text { - if } Z_{n}^{j}=0, j \in O_{n} \\
\text { - if } Z_{n}^{j}>0, j \in I_{n}\end{array}$} \\
\hline \multicolumn{2}{|c|}{ 3.2. process $O_{n}$ and $I_{n}$ separately: } \\
\hline$\forall j \in O_{n}:$ & $\forall j \in I_{n}:$ \\
\hline put $F_{n}^{j}=\mathrm{e}^{-\zeta \delta t} F_{n+1}^{j}$ & $\begin{array}{l}\text { - regress } C_{n}^{j}=\mathrm{e}^{-\zeta \delta t} F_{n+1}^{j} \text { on } 1, \\
S \text { and } S^{2} \text { to obtain a 2-degree } \\
\text { polynom } \hat{C}_{n}(S) \\
\text { - put } F_{n}^{j}=\max \left\{Z_{n}^{j}, \hat{C}_{n}(S)\right\} \\
\text { - if } Z_{n}^{j}>\hat{C}_{n}(S), \text { then } n \text { is the } \\
\text { new investment date, so put } \\
F_{m}^{j}=0 \quad \forall m>n\end{array}$ \\
\hline
\end{tabular}
theorem (see [15], Theorem 9.4.5.), we build by recursion a new Brownian motion $\left(W_{t}\right)$ under $\mathbb{P}$, such that $W_{t}=W_{t}^{\mathbb{Q}}-\int_{0<s<t} \theta\left(s, W_{s}\right) d s$.
}

TABLE I

DECISION TREE ALGORITHM

Let us denote by $O_{n}$ the set of the $j$ such that $Z_{n}^{j}=0$, and by $I_{n}$ the set of the $j$ such that $Z_{n}^{j}>0$. A summary of the whole algorithm is presented in Table I.

\section{Option premium}

Averaging the $F_{0}^{j}$, and using (13) and the law of large numbers, we obtain the premium $\Pi_{0}$ of the option:

$$
\Pi_{0} \approx \frac{1}{N} \sum_{j=1}^{J} F_{0}^{j}
$$

\section{Expected investing time}

Investigating our decision tree, it can happen that for some $j$ we do not decide to invest before $t^{\star}$. Then we will be lead to invest between $t^{\star}$ and $T^{4}$. For such trajectories, we do not know when the investment takes place. Furthermore, even for the other trajectories, additional information between $t^{\star}$ and $T$ can be useful to adjust the value of the investment date. For these two reasons, we decide to simulate $S_{t}$ further until $T^{5}$. Thus we perform one more time a backward dynamic algorithm, that time between 0 and $T$, using:

$$
\left\{\begin{array}{ll}
Z(t)=\left(S_{t}-K(t)\right)^{+} & \text {if } t>t^{\star} \\
Z(t)=\left(S_{t}-K^{\star}\right)^{+} & \text {otherwise }
\end{array} .\right.
$$

Finally, we obtain for each of the $J$ trajectories a best investment date $T_{\text {inv }}^{j}$. If $T_{\text {inv }}^{j}>t^{\star}$, it means that we have invested without exercising the original option, whereas if $T_{\text {inv }}^{j} \leq t^{\star}$, it means that we have exercised the option. Averaging the $T_{\text {inv }}^{j}$, we obtain the expected investing time

\footnotetext{
${ }^{4}$ Note that in theory, it could happen that we never decide to invest, even after $T$. However, given the deterministic trend of the demand, this would mean that $W_{t}$ remains extremely low. Considerations on the Brownian motion (see appendix VII-D) ensure that in practice it will not happen.

${ }^{5}$ That is the reason why we chose $\mathbb{Q}$ instead of $\mathbb{Q}^{\star}$.
} 
under the risk-neutral probability $\mathbb{E}_{\mathbb{Q}}\left[T_{\text {inv }}\right]$. But for us, it is more relevant to calculate $\mathbb{E}_{\mathbb{P}}\left[T_{\text {inv }}\right]$. Using (12), we obtain:

$$
\mathbb{E}_{\mathbb{P}}\left[T_{\text {inv }}\right]=\mathbb{E}_{\mathbb{Q}}\left[\frac{T_{\text {inv }}}{L_{T}}\right] \approx \frac{1}{N} \sum_{j=1}^{J} \frac{T_{\text {inv }}^{j}}{L_{T}^{j}} .
$$

\section{Numerical RESUlts}

In order to illustrate our algorithm, we applied it using the free simulator Scilab (see [18]). We considered a HSDPA pure data network with a random growing demand, as described in section II-A. We used the following parameters for our computation:

- the investment can take place until $T=150$ days.

- the equipment can be purchased at the initial price $K_{0}=300000 \$$, and its price decreases with a rate $\epsilon$ of $50 \%$ per year.

- the actualization rate $\zeta$ is fixed to $5 \%$ per year.

- the traffic demand starts at $x_{0}=1.2 \mathrm{Mbit} / \mathrm{sec} / \mathrm{cell}$, and increases with a drift fixed to $\alpha=0.54 \%$ per day. Its volatility is fixed to $0.01 \mathrm{day}^{-1 / 2}$. Its maximal value is fixed to $X_{\max }=3 \mathrm{Mbit} / \mathrm{sec} / \mathrm{cell}$ before the investment, and to $X_{\max }^{\prime}=8 \mathrm{Mbit} / \mathrm{sec} / \mathrm{cell}$ after the investment.

- the data transfer price is fixed to $\delta=0.1 \$ / \mathrm{Mbit}$.

- we take a satisfaction parameter of $\beta=0.7 \mathrm{Mbit} / \mathrm{sec} / \mathrm{cell}$.

- we simulate 10000 different trajectories of the asset.

\section{A. Option's price}

On Figure (3), we represent the price of the option versus $t^{\star}$. Recall that the price is obtained with equation (13), where $t^{\star}$ implicitly appears in function $Z$ (see equation (7)). It appears that the price increases with $t^{\star}$. This was expected, since the longer the option's maturity is, the higher the risk for the bank is, and then the more expensive the option is.

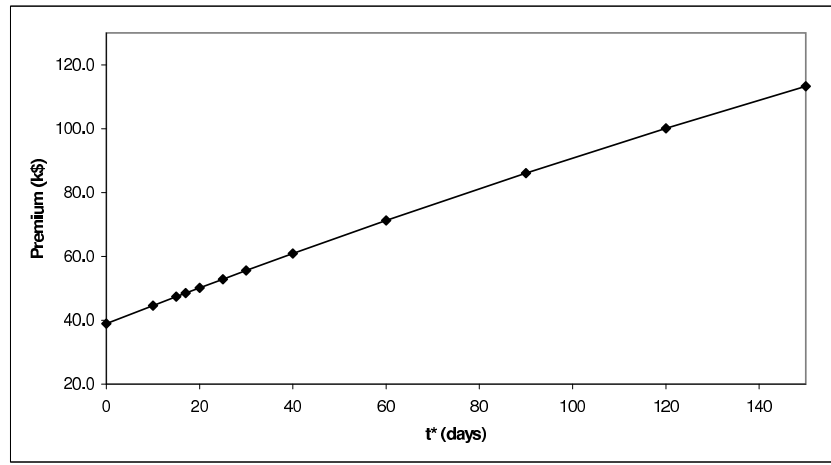

Fig. 3. Price of the option.

\section{B. Investment date}

On Figure (4), we represent the investment date versus $t^{\star}$. Recall that the date is obtained with equation (16), where $t^{\star}$ appears in generalized function $Z$ (see equation (15)), and may be prior to the investment's date. It appears that the investment date is very low for higher values of $t^{\star}$. This happens because $K^{\star}$ is very low, thus it is all the more interesting to invest

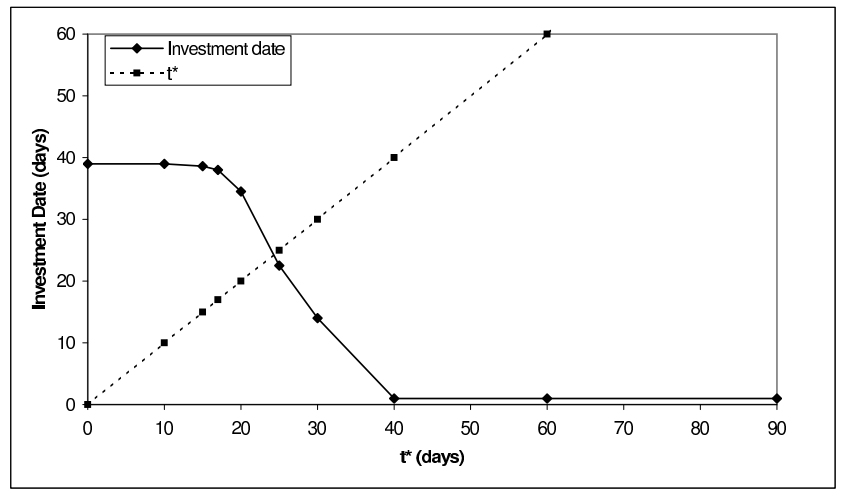

Fig. 4. Investment date.

early. As $K^{\star}$ decreases slowly toward 0 , the investment date decreases accordingly until reaching 0 for $t^{\star}=\infty$.

However, the lower the option's maturity is, the later the investment takes place. The investment date may even be later than $t^{\star}$, and the option is never exercised. This can be explained as follows: when $t^{\star}$ is low, $K^{\star}$, the equipment's exercise price, is quite high. Thus, the option is not really interesting. Rapidly the equipment's real price will sink under $K^{\star}$, and within that short period it is better to take the risk of waiting.

\section{CONCLUSION}

In this work, we proposed a model for risk hedging when dealing with investment under uncertainty in telecommunication networks. In such a case, the risk comes from the random evolution of the demand, possibly resulting in unexpected explosions of the traffic leading to network saturation. To hedge against this risk, the operator would buy an option from some financial parts that gives him the right but not the obligation of buying equipments at a given price, until a maturity date. We calculate, using backward dynamic programming and a least square approach, the premium of the option and the expected investment date. Our results show that the option price increases with the exercise date, whereas the mean investment date sinks. As a future work, we aim at considering the case where multiple investments are possible (in a telecommunication context, the multiple options may be adding more than one band, or implementing a more efficient technology, e.g. forthcoming 3G LTE systems).

\section{APPENDIX}

The purpose of this technical appendix is to prove that the mathematical conditions for applying a risk neutralization approach to price the American option are fulfilled. Precisely, there will be three main steps: 1- study the regularity of the function $v(t, x)$ from which the underlying asset is derived, and give a differential equation checked by its derivatives, 2deduce from Itō's lemma applied to $v$ the expression of the market price of risk $\theta_{t}$, and 3- verify Novikov condition on $\theta_{t}$ thanks to numerical simulations. 


\section{A. An explicit expression for $v(t, x)$}

Expression (6) of the underlying asset can be re-stated as follows:

$$
S_{t}=v\left(t, W_{t}\right),
$$

where we have introduced the function:

$$
v(t, x)=\mathbb{E}\left[\int_{t}^{T} \phi\left(t, s, W_{s}\right) d s \mid W_{t}=x\right]
$$

with:

$$
\phi(t, s, w)=\mathrm{e}^{-\zeta(s-t)}\left(\pi^{\prime}(s, w)-\pi(s, w)\right) .
$$

In this section, we aim at giving a fully explicit expression for the function $v(t, x)$, in order to study its properties in the following of the annexe. For this purpose, let us first swap sum and expectation in the expression of $v^{6}$. We obtain:

$$
v(t, x)=\int_{t}^{T} \mathbb{E}\left[\phi\left(t, s, x+W_{s}-W_{t}\right) \mid W_{t}=x\right] d s,
$$

and, since the increments of $W_{s}$ are independent:

$$
\begin{aligned}
v(t, x) & =\int_{t}^{T} \mathbb{E}\left[\phi\left(t, s, x+W_{s}-W_{t}\right)\right] d s \\
& =\int_{0}^{T-t} \mathbb{E}\left[\phi\left(t, t+s, x+W_{t+s}-W_{t}\right)\right] d s .
\end{aligned}
$$

Let us introduce another two functions:

$$
f(t, s, w)=\phi(t, t+s, w)
$$

and:

$$
u(t, s, x)=\int_{\mathbb{R}} f(t, s, w) g(x, s, w) d w,
$$

where $g(x, s,$.$) is the Gaussian density with mean x$ and variance $s$. We finally get:

$$
v(t, x)=\int_{0}^{T-t} u(t, s, x) d s .
$$

\section{B. Regularity and differential equation for $v$}

Let us first recall that:

$$
\left\{\begin{array}{l}
f(t, s, w)=\mathrm{e}^{-\zeta s}\left(\pi^{\prime}(t+s, w)-\pi(t+s, w)\right) \\
g(x, s, w)=\frac{1}{\sqrt{2 \pi s}} \mathrm{e}^{-\frac{(w-x)^{2}}{2 s}}
\end{array}\right.
$$

Lemma 1: $\pi(s, w)$ and $\pi^{\prime}(s, w)$ are $C^{\infty}(] 0,+\infty[\times \mathbb{R})$, bounded, with bounded derivatives

Proof: let us prove the property with $\pi^{1}$ for example.

- First we prove that $\pi$ is $C^{\infty}(] 0,+\infty[\times \mathbb{R})$. For all $\left.s_{0} \in\right] 0,+\infty\left[\right.$, let us introduce $w_{0}=h\left(s_{0}\right)$, the number such that $X\left(s_{0}, w_{0}\right)=X_{\max }$ :

$$
\sigma w_{0}=\log \left(X_{\max } / x_{0}\right)-\left(\alpha-\sigma^{2} / 2\right) s_{0}
$$

The points $\left(s_{0}, w_{0}\right)$ define a line $\Delta$ (see Fig. 5). Let us also introduce the two subsets of $] 0,+\infty[\times \mathbb{R}$ :

$$
\left\{\begin{array}{l}
\Omega_{1}=\{(s, w) / w<h(s)\} \\
\Omega_{2}=\{(s, w) / w \geq h(s)\}
\end{array}\right.
$$

\footnotetext{
${ }^{6}$ This is possible because $\phi$ is positive, since $0 \leq \pi \leq \pi^{\prime}$
}

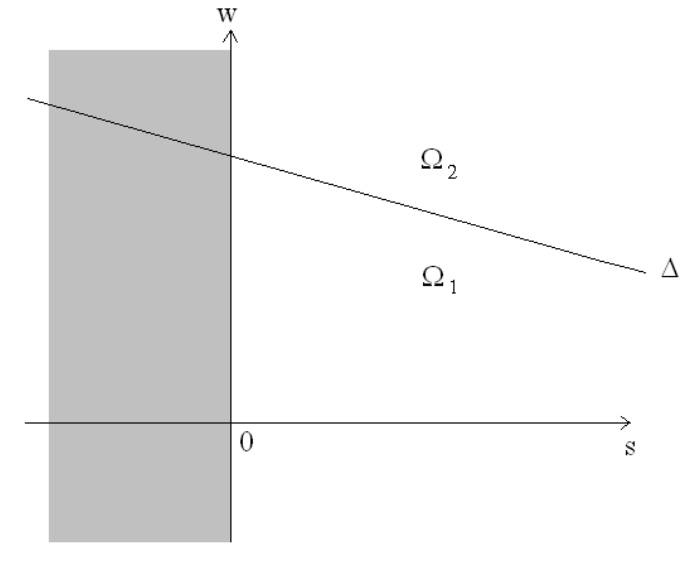

Fig. 5. Partition of the plane into two subsets $\Omega_{1}$ and $\Omega_{2}$.

These two subsets are situated respectively under and above $\Delta$. On $\Omega_{1}, X(s, w)<X_{\max }$ and we have:

$$
\pi(s, w)=\delta x_{0} \mathrm{e}^{\left(\alpha-\frac{\sigma^{2}}{2}\right) s+\sigma w} \mathrm{e}^{-\beta /\left(X_{\max }-x_{0} \mathrm{e}^{\left(\alpha-\frac{\sigma^{2}}{2}\right) s+\sigma w}\right)},
$$

and on $\Omega_{2}, X(s, w) \geq X_{\max }$ and $\pi=0$.

On $\Omega_{1}$, since:

$$
\left\{\begin{array}{l}
\frac{\partial}{\partial s} X=\left(\alpha-\sigma^{2} / 2\right) X \\
\frac{\partial}{\partial w} X=\sigma X
\end{array},\right.
$$

one can show by recursion over $n=p+q$ that the derivatives of $\pi$ can be written:

$$
\frac{\partial^{n}}{\partial s^{p} \partial w^{q}} \pi=\frac{P_{p, q}(X)}{\left(X_{\max }-X\right)^{2 n}} \mathrm{e}^{-\beta /\left(X_{\max }-X\right)}
$$

where $P_{p, q}$ is a polynom. Hence the denominator is counterbalanced by the second exponential term in the expression of the derivative of $\pi$, so that the derivatives all tend to 0 in the neighborhood of $\Delta$ and the transition between $\Omega_{1}$ and $\Omega_{2}$ is $C^{\infty}$.

- Secondly we prove that each derivative of $\pi$ is bounded. Expression (19) is a continuous function of $X$ on $\left[0, X_{\max }\right.$, and is also continuous at $X_{\max }$. Hence, it is bounded for $X \in\left[0, X_{\max }\right]$. Since:

$$
\left.\left.X\left(\Omega_{1}\right)=\right] 0, X_{\max }\right] \subset\left[0, X_{\max }\right],
$$

this achieves the proof.

Lemma 2: for any two compact sets $C \subset] 0,+\infty\left[, C^{\prime} \subset \mathbb{R}\right.$, we have the following upper bounds:

$$
\begin{array}{ll}
\forall s \in C, & \left|\frac{\partial^{i} g}{\partial s^{i}}\right| \leq \varphi_{x, C}^{i}(w) \\
\forall x \in C^{\prime}, & \left|\frac{\partial^{i} g}{\partial x^{i}}\right| \leq \psi_{s, C^{\prime}}^{i}(w)
\end{array}
$$

where $\varphi_{x, C}^{i}$ and $\psi_{s, C^{\prime}}^{i}$ are summable over $\mathbb{R}$.

Proof: we only prove the first upper bound, the second one is exactly similar. One can show by recursion that $\frac{\partial^{i} g}{\partial s^{i}}$ can be 
written:

$$
\frac{\partial^{i} g}{\partial s^{i}}=g(x, s, w) \sum_{k=0}^{d_{i}} a_{i, k}(s) w^{k}
$$

where each $a_{i, k}$ varies continuously with $s$. We want to bound (20) when $s$ varies within a compact set $C=[a, b] \subset] 0,+\infty[$. Since:

$$
\left|\frac{\partial^{i} g}{\partial s^{i}}\right| \leq g(x, s, w) \sum_{k=0}^{d_{i}}\left|a_{i, k}(s)\right||w|^{k},
$$

we deduce:

$$
\left|\frac{\partial^{i} g}{\partial s^{i}}\right| \leq \frac{1}{\sqrt{2 \pi a}} \mathrm{e}^{-\frac{(w-x)^{2}}{2 b}} \sum_{k=0}^{d_{i}}\left(\max _{s \in[a, b]}\left|a_{i, k}(s)\right|\right)|w|^{k},
$$

from which the first upper bound is immediate.

Lemma 3: $f$ is $C^{\infty}$ w.r.t. each of its variables $t \in[0, T$, $s \in] 0, T-t[, w \in \mathbb{R}$, and its derivatives are bounded.

Proof: this comes directly from Lemma 1. In particular, there exist constants $K_{i}$ and $K_{i}^{\prime}$ so that:

$$
\left|\frac{\partial^{i} f}{\partial t^{i}}\right| \leq K_{i} \quad, \quad\left|\frac{\partial^{i} f}{\partial s^{i}}\right| \leq K_{i}^{\prime}
$$

Lemma 4: $u$ is $C^{\infty}$ w.r.t. each of its variables $t \in[0, T$, $s \in] 0, T-t\left[, x \in \mathbb{R}\right.$. For any compact set $C^{\prime} \subset \mathbb{R}$ :

$$
\begin{aligned}
& \left|\frac{\partial^{i} u}{\partial t^{i}}\right| \leq K_{i} \\
& \left|\frac{\partial^{i} u}{\partial x^{i}}\right| \leq K_{i, C^{\prime}}, \forall x \in C^{\prime}
\end{aligned}
$$

and the following differential equation is verified by $u$ :

$$
u_{x x}^{\prime \prime}=2\left(u_{s}^{\prime}-\int_{\mathbb{R}} f_{s}^{\prime} g\right)
$$

Proof: using Lemma 3, we obtain:

$$
\left|\frac{\partial^{i}}{\partial t^{i}}(f g)\right|=\left|\frac{\partial^{i} f}{\partial t^{i}} g\right| \leq K_{i} g
$$

Then, the derivability of $u$ w.r.t. $t$ and the first upper bound immediately come from expression (17) and the differentiation under the integral sign theorem.

Using Lemmas 2 and 3, we obtain (for any $x \in C^{\prime}$ ):

$$
\left|\frac{\partial^{i}}{\partial x^{i}}(f g)\right|=\left|f \frac{\partial^{i} g}{\partial x^{i}}\right| \leq K_{0} \psi_{s, C^{\prime}}^{i}(w)
$$

Since $\psi_{s, C^{\prime}}^{i}$ is summable over $\mathbb{R}$, we deduce the derivability of $u$ w.r.t. $x$, along with the second upper bound, taking:

$$
K_{i, C^{\prime}}=K_{0} \int_{\mathbb{R}} \psi_{s, C^{\prime}}^{i}(w) d w
$$

Each derivative $\frac{\partial^{i}}{\partial s^{i}}(f g)$ is a sum of terms which can be written:

$$
\frac{\partial^{p} f}{\partial s^{p}} \cdot \frac{\partial^{q} g}{\partial s^{q}}(p+q=i)
$$

From Lemma 2 (taking $C=[0, T]$ ) and Lemma 3, we get:

$$
\left|\frac{\partial^{p} f}{\partial s^{p}} \cdot \frac{\partial^{q} g}{\partial s^{q}}\right| \leq K_{p}^{\prime} \varphi_{x,[0, T]}^{q}(w),
$$

which is integrable over $\mathbb{R}$. Hence, $u$ is $C^{\infty}$ w.r.t. $s$.

By deriving under the integral sign, we have:

$$
\begin{array}{ll}
u_{x x}^{\prime \prime}(t, s, x)= & \int_{\mathbb{R}} f(t, s, w) g_{x x}^{\prime \prime}(x, s, w) d w \\
u_{s}^{\prime}(t, s, x)= & \quad \int_{\mathbb{R}} f(t, s, w) g_{s}^{\prime}(x, s, w) d w \\
+ & \int_{\mathbb{R}} f_{s}^{\prime}(t, s, w) g(x, s, w) d w,
\end{array}
$$

(splitting into two sums is allowed since $f_{s}^{\prime} g$ is summable). Thanks to the heat equation verified by the Gaussian kernel:

$$
g_{s}^{\prime}=\frac{1}{2} g_{x x}^{\prime \prime}
$$

we finally get third assertion of Lemma 4:

$$
\begin{aligned}
u_{x x}^{\prime \prime} & =2 \int_{\mathbb{R}} f(t, s, w) g_{s}^{\prime}(x, s, w) d w \\
& =2\left(u_{s}^{\prime}-\int_{\mathbb{R}} f_{s}^{\prime}(t, s, w) g(x, s, w) d w\right) .
\end{aligned}
$$

Lemma 5: $v$ is $C^{\infty}$ w.r.t. each of its variables, $t \in[0,+\infty[$ and $x \in \mathbb{R}$, and $v_{t}^{\prime}+\frac{1}{2} v_{x x}^{\prime \prime}=-\phi(t, t, x)+\zeta v$.

Proof: the regularity of $v$ is a direct consequence of equation (18) and Lemma 4. The differential equation checked by $v$ is obtained as follows:

$$
\begin{aligned}
v_{t}^{\prime} & =\int_{0}^{T-t} u_{t}^{\prime} d s-u(t, T-t, x) \\
& =\int_{0}^{T-t} \int_{\mathbb{R}} f_{t}^{\prime} g d w d s-u(t, T-t, x) \\
& =\int_{0}^{T-t} \int_{\mathbb{R}}\left(f_{s}^{\prime} g+\zeta f g\right) d w d s-u(t, T-t, x) \\
& =\int_{0}^{T-t}\left(u_{s}^{\prime}-\frac{1}{2} u_{x x}^{\prime \prime}\right) d s+\zeta v-u(t, T-t, x) \\
& =u(t, T-t, x)-\lim _{s \rightarrow 0} u-\frac{1}{2} v_{x x}^{\prime \prime}+\zeta v-u(t, T-t, x) .
\end{aligned}
$$

$f(t, ., w)$ being continuous at point $s=0$, we have $\lim _{s \rightarrow 0} u(t, s, x)=\int_{\mathbb{R}} \phi(t, t, w) g(x, 0, w) d w$ where the term $g(x, 0, w)$ has to be understood as the Dirac distribution at $x$. Therefore:

$$
v_{t}^{\prime}=-\phi(t, t, x)-\frac{1}{2} v_{x x}^{\prime \prime}+\zeta v
$$

Lemma 6: $\frac{\partial v}{\partial x}(\mathrm{t}, \mathrm{x})$ is null only on a curve $x=x(t)$.

Proof: this lemma will not be rigorously proved, but instead inferred from numerical simulation of the surface $\frac{\partial v}{\partial x}(t, x), t \in[0, T[, x \in \mathbb{R}$. Fig. 6 shows this surface. Clearly, one can observe that on the left side of the red line, $\frac{\partial v}{\partial x}$ goes to zero only on a curve $x(t)$. What can be proved analytically is that $\frac{\partial v}{\partial x}(t, x)<0$ on the right side of the line, where paradoxically the surface is very close to zero. Let us write $\frac{\partial v}{\partial x}$ in a new way: 


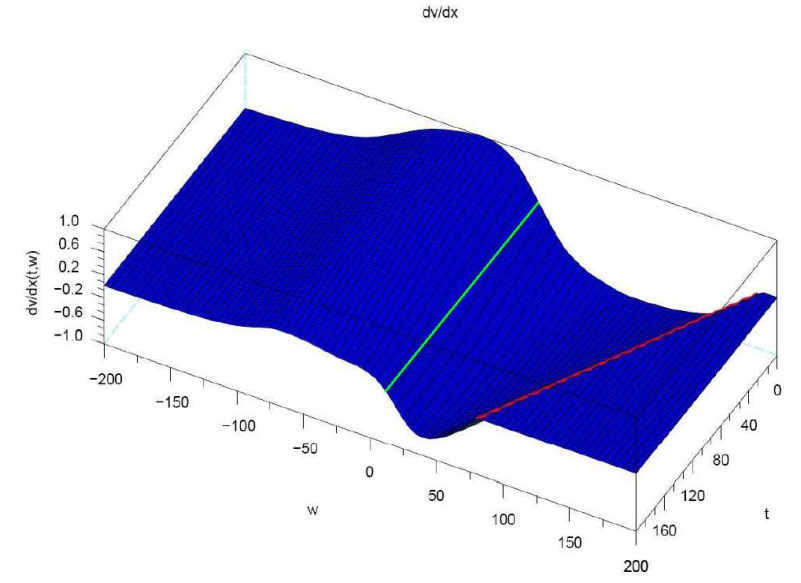

Fig. 6. The surface $\partial v / \partial x$ (we kept the previous parameters). On the right of the red line, we show in Lemma 6 that it is non null. On the left, we see that it is null only on the green line.

$$
\begin{aligned}
\frac{\partial v}{\partial x}(t, x) & =\int_{0}^{T-t} \int_{\mathbb{R}} f(t, s, w) \frac{\partial g}{\partial x}(x, s, w) d w d s \\
& =\int_{0}^{T-t} \int_{\mathbb{R}} f(t, s, w) \frac{w-x}{s} g(x, s, w) d w d s \\
& =\int_{0}^{T-t} \mathbb{E}\left[\phi\left(t, t+s, x+\bar{W}_{s}\right) \frac{\bar{W}_{s}}{s}\right] d s
\end{aligned}
$$

where $\bar{W}_{s}$ is a standard Brownian motion. $\phi\left(t, t+s, x+\bar{W}_{s}\right)$ is always positive, and null iff $t+s \geq a\left(x+\bar{W}_{s}\right)+b$, where we have introduced two coefficients:

$$
a=-\frac{\sigma}{\alpha-\sigma^{2} / 2} \quad, \quad b=\frac{\log \left(X_{\max }^{\prime} / x_{0}\right)}{\alpha-\sigma^{2} / 2} .
$$

Hence:

$$
\begin{aligned}
\frac{\partial v}{\partial x}(t, x)= & \int_{0}^{T-t} \mathbb{E}\left[\phi\left(t, t+s, x+\bar{W}_{s}\right) \frac{\bar{W}_{s}}{s} \mathbf{1}_{\left\{t+s<a\left(x+\bar{W}_{s}\right)+b\right\}}\right] d s \\
= & \int_{0}^{T-t} \mathbb{E}\left[\phi\left(t, t+s, x+\bar{W}_{s}\right) \frac{\bar{W}_{s}}{s} \mid \bar{W}_{s}<\frac{t+s-b}{a}-x\right] \\
& \quad \times \mathbb{P}\left(t+s<a\left(x+\bar{W}_{s}\right)+b\right) d s
\end{aligned}
$$

If $\frac{t-b}{a}-x \leq 0$, it is immediate to obtain $\frac{\partial v}{\partial x}(t, x)<0$. The line $\frac{a}{a}-x=0$ being precisely the red line of Fig. 6 , we have the result.

\section{Expression of the risk premium $\theta_{t}$}

In this section, we justify the existence of the market price of risk $\theta_{t}$ and deduce its expression from Itō's lemma and the differential equation checked by $v$. Itō's lemma holds since $v$ is regular. It gives:

$$
d S_{t}=\left[\frac{\partial v}{\partial t}\left(t, W_{t}\right)+\frac{1}{2} \frac{\partial^{2} v}{\partial x^{2}}\left(t, W_{t}\right)\right] d t+\frac{\partial v}{\partial x}\left(t, W_{t}\right) d W_{t}
$$

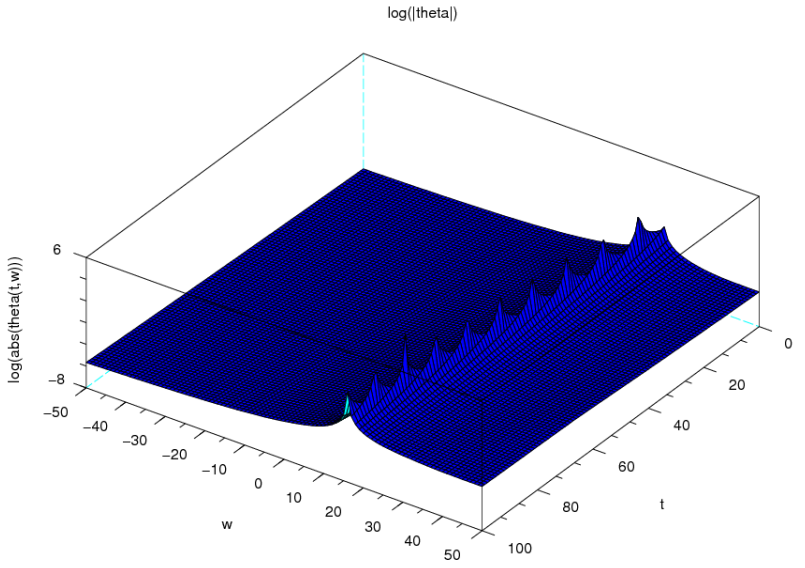

Fig. 7. Repartition of the peaks of $\theta(t, w)$. They all lie on the green line represented on Fig. 6. Normally, they should form a continuous crest, but due to discretization they show an uneven behavior.

Provided $X_{\max }^{\prime}>X_{\max }$, we have $v(t, x)>0$ for any $(t, x)$. Hence $S_{t}>0$ and we can write:

$$
\frac{d S_{t}}{S_{t}}=\frac{1}{v}\left(\frac{\partial v}{\partial t}+\frac{1}{2} \frac{\partial^{2} v}{\partial x^{2}}\right) d t+\frac{1}{v} \frac{\partial v}{\partial x} d W_{t} .
$$

By identifying this equation with the dynamics of the underlying asset (8), we get the expression of the expected total return on the asset $\mu_{t}$ and the volatility $\kappa_{t}$ :

$$
\left\{\begin{array}{l}
\mu_{t}=\frac{1}{v}\left(\frac{\partial v}{\partial t}+\frac{1}{2} \frac{\partial^{2} v}{\partial x^{2}}\right) \\
\kappa_{t}=\frac{1}{v} \frac{\partial v}{\partial x}
\end{array} .\right.
$$

Lemma 6 ensures that $\kappa_{t} \neq 0$ a.s., therefore the market price of risk $\theta_{t}$ is well defined, and:

$$
\theta_{t}=\left(\frac{\partial v}{\partial x}\right)^{-1}\left(\frac{\partial v}{\partial t}+\frac{1}{2} \frac{\partial^{2} v}{\partial x^{2}}-\zeta v\right)
$$

The final expression for $\theta_{t}$ is a consequence of Lemma 5:

$$
\theta_{t}=-\frac{\phi\left(t, t, W_{t}\right)}{\frac{\partial v}{\partial x}\left(t, W_{t}\right)}=-\frac{\pi^{2}\left(t, W_{t}\right)-\pi^{1}\left(t, W_{t}\right)}{\frac{\partial v}{\partial x}\left(t, W_{t}\right)} .
$$

\section{Novikov's condition}

Now we have to prove that:

$$
\mathbb{E}\left[\exp \left(\frac{1}{2} \int_{0}^{T} \theta_{t}^{2} d t\right)\right]<+\infty
$$

Actually, is $\int_{0}^{T} \theta_{t}^{2} d t$ even finite ? The question is relevant, because Lemma 6 shows that on a certain line, $\frac{\partial v}{\partial x}$ is null, and so $\theta(t, w)$ is infinite (see Fig. 7).

Now, could a trajectory $\left(W_{t}\right)$ come close to this line during a time long enough so that:

$$
\mathbb{E}\left[\exp \left(\frac{1}{2} \int_{0}^{T} \theta_{t}^{2} d t\right)\right]=+\infty ?
$$


Here we use a result of El Karoui and Gobet (see [15], Proposition 1.3.8.):

$$
\mathbb{P}\left(\sup _{t \leq T}\left|W_{t}\right| \geq c\right) \leq 2 \mathbb{P}\left(\left|W_{T}\right| \geq c\right),
$$

which tends to 0 extremely rapidly when $c \rightarrow \infty$. Hence, if we choose correctly our parameters so that the critic line lies far enough from the line $w=0$, the probability to reach it during the experiment will be extremely low. Then, in practice, we will consider that $\theta_{t}$ remains almost surely bounded by a constant $\hat{\theta}$; and a fortiori, Novikov's condition will be verified, since we will have:

$$
\mathbb{E}\left[\exp \left(\frac{1}{2} \int_{0}^{T} \theta_{t}^{2} d t\right)\right] \leq \mathrm{e}^{T \hat{\theta}^{2} / 2}
$$

\section{E. Regressing a set of points on a 2-degree polynom}

Given a set of points $\left(x_{i}, y_{i}\right)_{1 \leq i \leq n}$ in $\mathbb{R}^{2}$, the aim of the section is to find three real numbers $a, b, c$, such that

$$
\sum_{i=1}^{n}\left|y_{i}-P_{a, b, c}\left(x_{i}\right)\right|^{2}
$$

is minimal, where:

$$
P_{a, b, c}(x)=a x^{2}+b x+c .
$$

After deriving w.r.t. $a, b$ and $c$, we obtain respectively:

$$
\begin{cases}a \sum x_{i}^{4}+b \sum x_{i}^{3}+c \sum x_{i}^{2} & =\sum x_{i}^{2} y_{i} \\ a \sum x_{i}^{3}+b \sum x_{i}^{2}+c \sum x_{i} & =\sum x_{i} y_{i} \\ a \sum x_{i}^{2}+b \sum x_{i}+c & =\sum y_{i} .\end{cases}
$$

Has (21) a solution? Let us consider the 4 vectors of $\mathbb{R}^{n}$ :

$$
x^{2}=\left(\begin{array}{c}
x_{1}^{2} \\
\vdots \\
x_{n}^{2}
\end{array}\right) x=\left(\begin{array}{c}
x_{1} \\
\vdots \\
x_{n}
\end{array}\right) \mathbf{1}=\left(\begin{array}{c}
1 \\
\vdots \\
1
\end{array}\right) y=\left(\begin{array}{c}
y_{1} \\
\vdots \\
y_{n}
\end{array}\right) \text {, }
$$

then we can re-write the system as:

$$
\left(\begin{array}{ccc}
\left(x^{2} \mid x^{2}\right) & \left(x^{2} \mid x\right) & \left(x^{2} \mid \mathbf{1}\right) \\
\left(x \mid x^{2}\right) & (x \mid x) & (x \mid \mathbf{1}) \\
\left(\mathbf{1} \mid x^{2}\right) & (\mathbf{1} \mid x) & (\mathbf{1} \mid \mathbf{1})
\end{array}\right)\left(\begin{array}{l}
a \\
b \\
c
\end{array}\right)=\left(\begin{array}{c}
\left(x^{2} \mid y\right) \\
(x \mid y) \\
(\mathbf{1} \mid y)
\end{array}\right)
$$

It is equivalent to say that the vector $y-a x^{2}-b x-c$ is orthogonal to $\mathbf{1}, x$ and $x^{2}$. In other words, $a x^{2}+b x+c$ is the orthogonal projection of $y$ on $\operatorname{Vect}\left(1, x, x^{2}\right)$, and thus we are sure that (21) has a solution.

Is this solution unique ? If $\mathbf{1}, x$ and $x^{2}$ were not independent, there would be three real numbers $u, v$ and $w$ such that:

$$
\forall i, \quad u+v x_{i}+w x_{i}^{2}=0 .
$$

- as soon as there are more than two different values of $x_{i}$, this is impossible, and so there is a unique solution.

- if $x_{i}$ takes only two different values, then the solution is not unique any more, but $\left(\mathbf{1}, x, x^{2}\right)$ has rank 2 . So we choose to regress $y$ on $\mathbf{1}$ and $x$ for example (and we find a line which intersects the centroids of the two corresponding subsets).

- if $x_{i}$ takes only one value, then $\left(1, x, x^{2}\right)$ has rank 1 . So we choose to regress $y$ on $\mathbf{1}$ (and we find the mean of the $\left.y_{i} \mathrm{~s}\right)$.

\section{REFERENCES}

[1] A.K. Dixit and R.S. Pindyck, Investment Under Uncertainty, Princeton University Press, 1994.

[2] L. Trigeorgis, Anticipated Competitive Entry and Early Preemptive Investment in Deferrable Projects, Journal of Economics and Business, 43(1991), 143-156.

[3] M. J. Brennan and E. S. Schwartz, Evaluating Natural Resource Investments, The Journal of Business, Vol. 58, No. 2. (Apr., 1985), pp. 135-157.

[4] J.-D. Saphores and M.G. Boarnet, Investing in Urban Transportation Infrastructure Under Uncertainty, 8th Annual International Conference Montreal Canada, June 17-19, 2004.

[5] L. Salahaldin and T. Granger, Investing in Sustainable Transport to Relieve Air Pollution under Population-Growth Uncertainty, 9th Annual International Conference on Real options, Paris, June 2005.

[6] A.A. van Benthem, G.J. Kramera and R. Ramerb, An options approach to investment in a hydrogen infrastructure, Energy Policy, 2005.

[7] A. C. Marco, The option value of patent litigation: Theory and evidence, Review of Financial Economics 14 (2005) 323-351.

[8] L. Salahaldin, A Numerical Algorithm for Decision-making in Sustainable Transport Projects Investment, 11th Annual International Conference on Real options, Berkeley, CA, June 2007.

[9] G. Fiuza de Braganca, K. Rocha and R. Rodrigues Moreira, Real Options and the Regulation of Brazilian Fixed-Line Telephone Operators: The Markup on the Cost of Capital, Annual Real options Conference, Rio De Janeiro, Brazil, 2008.

[10] G. Sick, Real Options and Facilities Access Regulation, Annual Real options Conference, Rio De Janeiro, Brazil, 2008.

[11] V. Tanguturi and F. Harmantzis, Migration to $3 G$ wireless broadband Internet and real options: The case of an operator in India, Telecommunications Policy, Volume 30, Issue 7, August 2006, Pages 400-419.

[12] Y. d'Halluin, P.A. Forsyth, K.R. Vetzal, Managing Capacity for Telecommunications Networks Under Uncertainty, IEEE/ACM transactions on networking, Vol. 10, No. 4, August 2002.

[13] Y. d'Halluin, P.A. Forsyth, K.R. Vetzal, Wireless Network Capacity Investment, European Journal on Operational Research 176 (2007) 584609.

[14] T. Bonald and A. Proutière, Wireless Downlink Data Channels: User Performance and Cell Dimensioning, ACM Mobicom 2003, April 2003.

[15] N. El Karoui, E. Gobet, Introduction au Calcul stochastique, première partie, Imprimerie de l'École Polytechnique, 2003.

[16] N. El Karoui, Introduction au Calcul stochastique, deuxième partie: finance, Imprimerie de l'École Polytechnique, 2003.

[17] N. Enderlé, X. Lagrange, User Satisfaction Models and Scheduling Algorithms for Packet-Switched Services in UMTS, IEEE VTC, 2003.

[18] C. Gomez (Ed.), Engineering and Scientific Computing with Scilab, ISBN: 978-0-8176-4009-5, 1999.

[19] M. Broadie, J.B. Detemple, Option Pricing: Valuation Models and Applications, Management Science, No. 50.

[20] F.A. Longstaff, E.S. Schwartz, Valuing American Options by Simulation: A Simple Least-Squares Approach, The Review of Financial Studies, Vol. 14, No. 1. 